\title{
Rollenspiele für Ober- und Chefärzte
}

\author{
In einem Pilot-Workshop in Luzern lernten Kaderärzte, wie sie ihre Mitarbeiter besser \\ betreuen und führen. Den Workshop initiierte das Schweizer Institut für ärztliche \\ Weiter- und Fortbildung gemeinsam mit dem Royal College of Physicians in London. \\ Das Feedback der Teilnehmer war so gut, dass es die Kurse regelmässig geben soll.
}

Felicitas Witte

Dr. med.,

freie Medizinjournalistin

\begin{abstract}
Die junge Assistenzärztin bricht gleich in Tränen aus. Sie steht vor dem Patientenzimmer, ihre Wangen sind vor Scham gerötet. Ihr Chef stellt eine Frage nach der anderen, es tönt wie beim Kreuzverhör vor Gericht. Eine Pflegerin eilt vorbei - hektischer Stationsalltag. Sie habe einen Fehler gemacht, ob ihr das denn nicht bewusst gewesen sei, donnert der Chefarzt. «Ja, schon, aber ...»- Die junge Ärztin kommt gar nicht dazu, ihren Satz zu beenden. Warum sie das denn gemacht habe, schimpft der Mediziner weiter. Stotternd versucht die Frau zu erklären. Doch der Arzt hört ihr kaum zu und sagt im Weggehen: «Das wird Konsequenzen haben.» Entsetzt schaut ihm die junge Frau nach.
\end{abstract}

\section{«Mit unseren Workshops wollen wir Ärzten Wissen und Fähigkeiten für den nicht-klinischen Bereich vermitteln.»}

Korrespondenz:

Schweizerische Ärztezeitung Farnsburgerstrasse 8 CH-4132 Muttenz

redaktion.saez[at]emh.ch

\section{Grosses Interesse am Kursangebot}

Man spürt förmlich ihre Verzweiflung, doch zum Glück ist alles nur gestellt. Die junge Frau und der Mann mit den grauen Haaren spielen Assistenzärztin und Chefarzt in einem Krankenhaus in Grossbritannien. Das Ganze ist ein Videoclip, dem 35 Schweizer Ärztinnen und Ärzte an diesem sommerlichen Septembertag gebannt folgen. Es ist der erste Tag einer Weiterbildung, der aus mehreren Workshops besteht. Hier können Ärzte in Weiterbildungsverantwortung lernen, wie sie ihre Mitarbeiter besser führen, wie man Probleme und Konflikte am besten löst und was zu einer guten Zusammenarbeit im Team beiträgt. Die Fortbildung ist ein Pilotprojekt, initiiert und organisiert vom Schweizerischen Institut für ärztliche Weiter- und Fortbildung SIWF in Zusammenarbeit mit dem Royal College of Physicians in London. «Leitende Ärzte haben immer öfter Schwierigkeiten, ihren Führungsaufgaben nachzukommen», sagt Werner Bauer, Präsident des SIWF. Das läge zum einen daran, dass wegen zunehmender Bürokratie weniger Zeit zur Verfügung stünde. «Ausserdem fällt es manchen schwer, ihre Führungsposition gegenüber kriti- schen jungen Kollegen wahrzunehmen.» Bei einem Besuch im Royal College vor zwei Jahren war Bauer fasziniert von den Weiterbildungen, die diese Institution anbietet. «Da dachte ich mir: So was bräuchten wir für unsere Ärzte auch!» Inhalt und Ablauf hatte er rasch mit den Organisatoren der Workshops abgesprochen, ein halbes Jahr später konnten sich Ärzte bewerben. «Das Interesse übertraf alle Erwartungen - innerhalb weniger Tage waren die Plätze ausgebucht», erzählt Bauer. Der Workshop bestand aus zwei eintägigen und einem zweitägigen Kurs. In den ersten beiden eintägigen Kursen konnte man effektive Strategien lernen, wie man am besten leistungsschwache Mitarbeiter unterstützt und wie man gut unterrichtet. Im dritten zweitägigen Kurs ging es allgemein darum, wie man eine gute Führungspersönlichkeit wird, unter anderem durch konstruktives Feedback, guten Umgang mit leistungsschwachen Mitarbeitern und Ausbau der eigenen Führungsqualitäten.

\section{Konkrete Lösungen für Probleme des Alltags}

«What did you think went wrong?», fragt David Perry nach dem ersten Videoclip in die Teilnehmerrunde. Zunächst in Zweierpaaren, später in der grossen Gruppe diskutieren die Teilnehmer lebhaft das Verhalten des britischen Chefarztes. «Ich wollte lernen, wie und in welcher Umgebung ich Mitarbeitergespräche am besten führen kann», sagt Frank Zimmermann, Chef-Radioonkologe am Unispital Basel. «Der Kurs hat mir hierbei sehr geholfen.» Viele reelle Beispiele aus der Praxis seien gebracht und diskutiert worden. «Wir wurden ständig angeregt, uns in die Rolle eines anderen zu versetzen und miteinander Probleme zu lösen - das war wirklich gut.» Einzig die Zeit für die Rollenspiele sei manchmal zu knapp gewesen. «Ich kann den Kurs unbedingt empfehlen am besten im letzten Jahr vor der Facharztprüfung, beziehungsweise bevor man eine Führungsposition einnimmt.» Zimmermann plant, den Workshop für seine Mitarbeiter zur Pflicht zu machen.

Schwierigen Mitarbeitern Feedback zu geben, findet Martin Beck, Chefarzt in der Orthopädie am Luzerner Kantonsspital, nach dem Kurs zwar immer noch schwierig. «Aber ich kenne jetzt Techniken, mit 
Interview mit Dr. med. Werner Bauer, Präsident des Schweizerischen Instituts für ärztliche Weiter- und Fortbildung SIWF die Organisatoren Kurse zum Thema «Teach the Teacher» auf der ganzen Welt anbieten und fand die Idee überzeugend: genau das, was wir suchen. Warum müssen wir erst einen Kurs neu erfinden, wenn das Royal College damit jahrelange Erfahrung hat?

Liessen Sie bei Inhalt und Struktur den Organisatoren freie Hand oder bestimmten Sie das mit?

Wir haben die Workshops gemeinsam geplant. Wir vom SIWF sagten, welche Inhalte uns wichtig sind. Die Experten des Royal College haben den Kurs dann auf unsere Bedürfnisse abgestimmt.

Der erste Kurs war ein voller Erfolg (siehe Artikel). Wird es die Workshops jetzt regelmässig geben?

Ja, wir sind schon dabei, die nächsten Kurse zu planen. Sie werden auch in Luzern stattfinden. Für die inhaltliche Gestaltung der Workshops werden wir uns die Rückmeldungen der Teilnehmer genau ansehen und die Kurse an Wünsche und Vorschläge anpassen. Wir wollen zum Beispiel die Themen der einzelnen Tage im voraus klarer definieren.

Der erste Kurs kostete nur eine Bearbeitungsgebühr von 50 Franken, den Rest übernahm das SIWF. Wird das so bleiben?

Das war leider nur für die Pilotkurse möglich. Wir rechnen mit ungefähr 250 Franken für einen Tag und 450 für zwei Tage. Wir bemühen uns aber, die Kosten in Grenzen zu halten. Wir möchten weiterbildenden Ärzten in der Schweiz etwas Exzellentes zu verträglichen Preisen anbieten - denn die Weiterbildner leisten enorm viel bei der Ausbildung des medizinischen Nachwuchses. denen ich die Situation entschärfen oder gar nicht erst eskalieren lassen kann.» Konkrete Lösungen für Probleme des Alltags habe der Kurs vermittelt, der Austausch mit den anderen Teilnehmern sei sehr konstruktiv gewesen. Er habe den Kurs vor allem gemacht, um besser unterrichten zu können. «Unser Nachwuchs muss gut ausgebildet werden», sagt Beck, «gut zu lehren, muss man aber lernen.»

\section{Auch Weiterbildner brauchen Unterstützung} «Mit unseren Workshops wollen wir Ärzten Wissen und Fähigkeiten für den nicht-klinischen Bereich vermitteln», sagt Winnie Wade, Mit-Organisatorin des Workshops und Direktorin für Weiterbildung am Londoner Royal College of Physicians. Alle Workshops werden gemeinsam von Ärzten und erfahrenen Pädagogen abgehalten. «Das ist der Schlüssel für den Erfolg unserer Programme.» Die Pädagogen vermitteln theoretische Hintergründe und praktische Tipps, die Ärzte bringen Erfahrung und Kenntnisse aus ihrer medizinischen Praxis mit. Das Royal College startete die Workshops vor über zwölf Jahren. «Damals gab es wenig, das Ärzte auf leitende Aufgaben vorbereitet», erzählt Wade. In den folgenden Jahren wurden die Kurse an die Bedürfnisse der Ärzte angepasst und weiterentwickelt. Inzwischen werden sie auf der ganzen Welt angeboten, unter anderem in Europa, den USA und Südasien. Sehr enthusiastisch und aufgeweckt seien die Schweizer Teilnehmer ge- wesen und hätten sich rege beteiligt. Sie hofft auf eine langfristige Partnerschaft mit dem SIWF und dass die Kurse vielen Ärzten bei ihrer Entwicklung helfen werden.

«Heutzutage werden viele Ärztinnen und Ärzte zu Oberärzten befördert, wenn sie noch ziemlich jung sind», sagt Simon Ritter, Oberarzt mit erweiterter Verantwortung in der Inneren Medizin am Triemlispital Zürich, «als «Weiterbildender in Weiterbildung〉 braucht man selbst noch Unterstützung und muss die Assistenzärzte gleichzeitig gut führen und weiterbilden.» Leider bleibe im Alltag dafür oft wenig Zeit. «Mir helfen jetzt die fünf Schritte des MikroskillsModells sehr, die wir im Kurs gelernt haben», sagt Ritter. Dabei fordert man den Studenten oder Assistenzarzt auf, sich auf eine Diagnose oder ein Behandlungskonzept festzulegen und seine Überlegungen zu begründen. Dann erklärt man die wichtigsten Aspekte zu der Erkrankung. Zum Schluss lobt man den

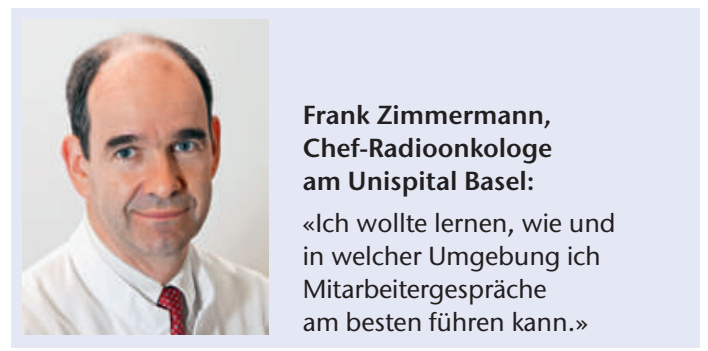




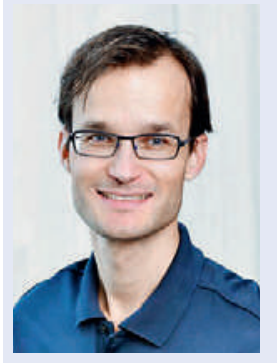

Simon Ritter, Oberarzt mit erweiterter Verantwortung in der Inneren Medizin am Triemlispital Zürich: «Als «Weiterbildender in Weiterbildung > braucht man selbst noch Unterstützung und muss die Assistenzärzte gleichzeitig gut führen und weiterbilden.»

jungen Mediziner für korrekte Handlungen und korrigiert seine Fehler. Dabei kritisiert man konstruktiv mit konkreten Verbesserungsvorschlägen. «Damit kann man das fokussierte und kritische Denken bei Studenten und Assistenten stärken, und sie lernen, wie man im Klinikalltag zu Entscheidungen kommt», sagt Ritter. Er habe ausserdem die Dynamik in kleinen Lerngruppen besser verstanden. «Im Studentenkurs kann ich jetzt viel besser auf die Bedürfnisse der Lernenden eingehen, statt starr nach einem Lehrkonzept vorzugehen.»

Der Kurs wurde durchgehend auf Englisch abgehalten, was den meisten kein Problem bereitete. «Ich hätte es aber angenehm gefunden, wenn der Kurs auf Deutsch gewesen wäre», sagt Ueli Zellweger, Chefarzt der Medizinischen Abteilung am Regionalspital Surselva. «Auch wenn ich das meiste verstanden habe, habe ich manchmal die Pointe eines Witzes oder Feinheiten in einem Gespräch nicht richtig mitbekommen.» Doch trotz der kleinen Sprachbarriere habe er Tipps und Hilfsmittel mitgenommen, die er in Zukunft einsetzen will. «Ich finde zum Beispiel den Vorschlag gut, Assistenzärzte durch nichtärztliche Mitarbeiter wie Pflegekräfte oder Physiothera-

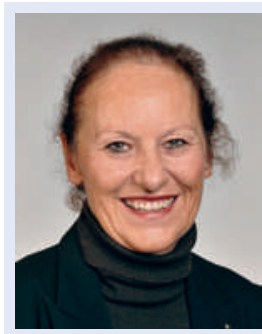

Elisabeth Bandi-Ott, Leiterin Lehre am Institut für Hausarztmedizin der Universität Zürich: «Assistenzärzte müssen im Sinne der Qualitätssicherung auch nach dem Staatsexamen weiter ausgebildet werden.»

peuten regelmässig und systematisch beurteilen zu lassen», sagt Zellweger. In einem kleinen Regionalspital sei es nicht immer einfach, den Nachwuchs gut auszubilden. «Die Herausforderung, einem leistungsschwachen Assistenzarzt seine mangelhafte Leistung zu kommunizieren und ihn dabei gleichzeitig zur Leistungssteigerung und Verbesserung zu motivieren, wird bleiben», sagt Zellweger. «Ich habe ge- merkt, dass regelmässigere Feedback-Gespräche notwendig sind.»

\section{Wunschliste für künftige Kurse}

Auch objektiv waren die Workshops ein voller Erfolg, wie die Auswertung der Fragebögen zeigt. Die meisten Teilnehmer fanden Inhalt und Aufbau der Kurse gut bis sehr gut, sie waren zufrieden und hatten Spass dabei. Der Kurs habe den Ankündigungen gut bis sehr gut entsprochen und sei relevant für die Praxis. Eine lange Wunschliste gibt es für zukünftige Kurse: Teilnehmer wünschen sich zum Beispiel zu lernen, wie man über-selbstbewusste Assistenten besser führt, wie man seine Zeit besser plant und einteilt oder wie man mit Krisen umgeht. «Die Rückmeldungen waren so positiv - den Kurs wird es nächstes Jahr wieder geben», sagt Bauer. Leider wird er dann mehr als die Be-

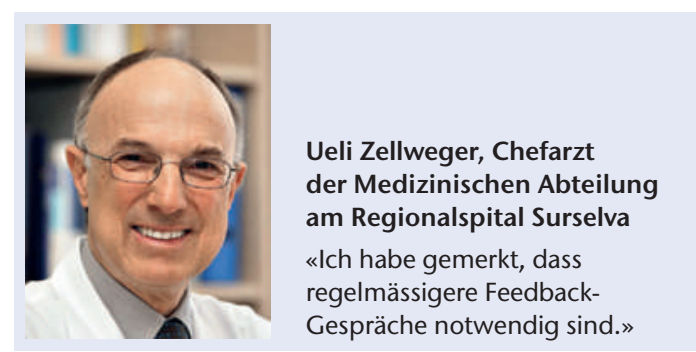

arbeitungsgebühr von 50 Franken kosten. «Ich hätte den Kurs trotzdem gemacht», sagt Elisabeth BandiOtt, Leiterin Lehre am Institut für Hausarztmedizin der Uni Zürich. «Assistenzärzte müssen im Sinne der Qualitätssicherung auch nach dem Staatsexamen weiter ausgebildet werden», sagt Bandi-Ott. Der Kurs vermittle dafür die richtigen Techniken. «Konstruktives Feedback ist eine der wichtigsten Grundlagen für gute Lehre.»

In einer zweiten Videosequenz sehen die Teilnehmer, wie es der britische Chefarzt richtig macht: Er bittet die Assistenzärztin zum Gespräch in einen ruhigen Raum und sagt Bescheid, dass man nicht stören solle. Ruhig hört er zu und stellt an den richtigen Stellen Zwischenfragen. Sie fühle sich in der neuen Stadt sehr alleine, erzählt die junge Frau, ausserdem leide sie unter schlimmen Schlafstörungen. Die vielen Nachtdienste machten sie völlig fertig. Vermutlich habe sie den Fehler deshalb gemacht. Der Chef empfiehlt, einen Hausarzt aufzusuchen und bespricht mit ihr, dass sie vorübergehend weniger Dienste machen kann. Er spricht aber auch den Fehler an und erklärt, was hätte passieren können. Erleichtert und froh verspricht die Ärztin, sich gleich um einen Hausarzt zu kümmern - und so einen Fehler wolle sie nie wieder machen. 